\title{
Eye Direction Based Safety Navigation System for Physically Challenged Person
}

\author{
Mouliraj. $V^{1^{*}}$, Mohanraj. $V^{2}$, Senthilkumar. $\mathbf{J}^{3}$, Suresh. $\mathbf{Y}^{4}$,Shanmugaraja.P ${ }^{5}$ \\ PG Scholar, Information Technology, Sona College of Technology, Salem, India ${ }^{1}$ \\ Professor, Information Technology, Sona College of Technology, Salem, India ${ }^{2,3,4}$ \\ Associate Professor, Information Technology, Sona College of Technology, Salem, India ${ }^{5}$
}

\begin{abstract}
This research papers include the eye direction based safety automated navigation system that implemented for the elderly and physically challenged people. The purpose of this navigation system is to avoid the assist required for the physically challenged people. This systems control the motorized wheelchair navigation depends on the eye pupil detection. By the image processing technique $(\mathrm{CNN})$, the sequential images have been capture via Bluetooth specs glass. The system navigate the user to desired directions such as move towards left, move towards right, move forward and stop. Additionally sensors are fixed in front of wheelchair to detect the objects to avoid the faulty navigation. A centralized wireless detector device is also made available in wheelchair for an emergency purpose. A raspberry pi model B is high speed detection kit controls the whole system. Another technique used in this navigation system speech to text conversion. Speech convert to text and then a connection established to raspberry pi model kit. So the system automatically moves by the users given the instruction.
\end{abstract}

Keywords: Bluetooth, Motorized Wheelchair, Eye Detection, Safety System, CNN

\section{INTRODUCTION}

Eye pupil detection is controlled to elderly and physically challenged people. The physically challenged people find it difficult to turn the wheel because great amount of energy is needed [1]. Presently, various types of eye based method are used for controlling the motorized wheelchair like EOG (Electrophysiology), a technique for measuring the corner retinal standing potential that is mainly derived from the (Retinal Pigment Epithelium) RPF and it changes in response to retinal illumination. The potential decreases for 8-10 min in darkness ECG, EEG based eye ball sensing method to decide the location from one place to another place using eye pupil moments depending on voltage and vibration [2]. The Convolution Neural Networks (CNN) have recently great success in the image processing concepts and it is used to classify the new cases based on extracted features in the neural networks. Image classification is an important role in the machine learning and image processing, which is common to many fields such as image retrieval and military automation. Neural networks that works on the basis of the structure and functions of a human brain. Like the human brain has interconnected artificial neurons that transmit data among each other and are called as node.

The neural networks consist of three layers input layer, hidden layer and output layer. The input layer is used to accept inputs in different forms. Hidden layer transform the inputs and do several calculations and feature extractions. The output layer produces the desired output. CNN accepts arrays of pixel values as input to the network. The hidden layer consists of several different layers which carry out feature extraction. Fully connected layer recognizes the object and image.

The convolution uses a filter matrix over the array of image pixels and performs convolution operation to obtain a convolved feature map. The next layer is the ReLU layer which introduce the non-linearity to the networks. It sets all negative pixels to zero and performs element wise operation the original image is scanned in multiple convolution and ReLU layers locating hidden features and pattern in the image. The image pixel value is 0 and 1 in the CNN convolution layer. Pooling layer reduces the dimensionality of the feature map [3].

Flattening is a method used on a pooled feature map to convert a long continuous linear vector. This process is called flattened. This flattened matrix goes through a fully connected layer to classify the image.

*Corresponding Author

Mouliraj.V ${ }^{1 *}$ E-Mail ID:mouli220@gmail.com 
Vol. 8, Issue 1, January 2019

\section{RELATED WORK}

In [4], the authors have compared the performance of unsupervised feature learning and transfer learning against simple patch initialization and random weight initialization within the same setup. They showed that pre-training helps to train CNNs from few samples and that the correct choice of the initialization scheme can push the network's performance by up to $41 \%$ compared to random initialization. Their result shows that pre-training systematically improves generalization capabilities when handling datasets with few samples.

In another research work by Norton online 2010 Malaysia for detection of cyber bullying states that Malaysian children spend an average of $19 \mathrm{hrs}$ a week on internet while that same also found that more than $80 \%$ of the children have been affected by negative comments and this is mainly done by their close friends or spouses. This work mainly focuses on the statistics of the cyber bullying [5].

The authors [6], have derived a new algorithm for convolution neural networks using wino grad's minimal filtering algorithm derived for network layers with 6 X6 as filter size for image recognition tasks. To reduce the complexity up to 4 times compared with direct convolution using small block sizes limited transform overhead and high computational intensity.

The authors [7], have said the autonomous behaviours an intelligent wheelchair platform this method is used to a shared control framework has different levels of autonomy allowing the human operator complete control of the chair at each level while ensuring the user's safety.

The authors [8], says the IR sensor based wheelchair system using raspberry pi for charcot marie-tooth. The work depends on the development of a wheelchair that can be a fully automatic navigation system. It provides flexible operation to choose different modalities to command the wheel chair, this method very useful to a people who can affected for this type disease. Patient can command the wheelchair based on eye blinks, eye movement. The wheelchair can operate like auto guided vehicle following IR sensor way.

The authors [9], says about Irish segment from eye image by applying the concept of overlapping blocks and fuzzy logic. Eye image is pre-processed and divided into a number of overlapping blocks in the form of a matrix. Mathematically proved by the mean, entropy and deviations to founded the blocks and these blocks are shaped to corresponding images.

\section{METHODOLOGY}

In this work, an improved method of eye detection using CNN algorithm has been presented. This automated navigation system is used to avoid the faulty navigation to a wheelchair root path and only detect on eye movement using CNN. $\mathrm{CNN}$ algorithm finds the accuracy of the eye movement in the image processing techniques. Raspberry pi 3 model b kit controls the used navigation system. This navigation system can be used by physically challenged people, elderly person and also blind person for safe navigation from one place to another.

A. Convolution neural networks(CNN): The convolution neural networks achieve about $95 \%$, matching human accuracy using distance between the vectors. L1 and L2 values are calculated based on the distance of the two vectors. Test image and training image are used to process pixel.

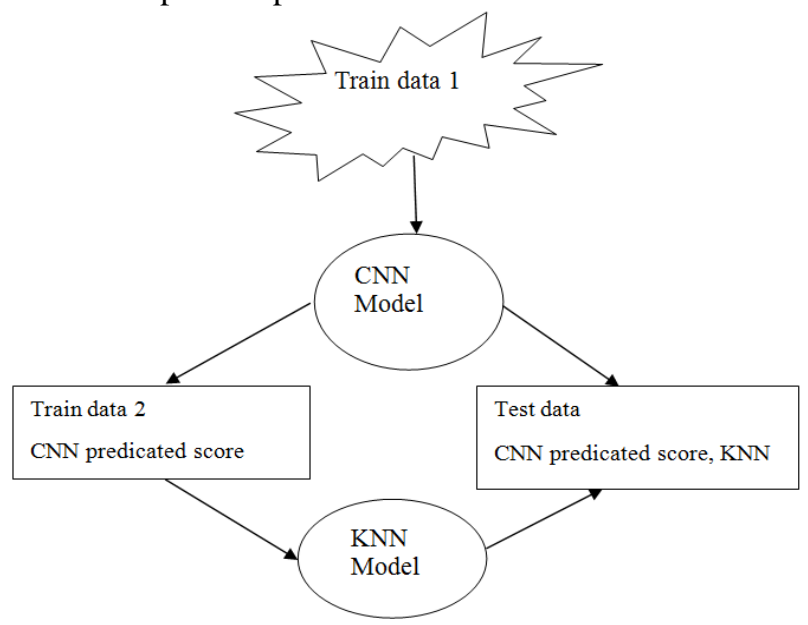

Fig. 1 methodology of predicate score in CNN and KNN

The following steps are involved:

- Split data.

- Image classification model and training data. 
Vol. 8, Issue 1, January 2019

- $\quad$ Predict second and third data.

- $\quad$ Make KNN model by second data's predicated score.

- $\quad$ Predict third data by the KNN model.

Fig. 1 shows that training and testing data in the $\mathrm{CNN}$ and $\mathrm{KNN}$ algorithm. In this algorithm the data is split into two types: training and test data, we have made CNN model by train data and predict train data itself and test data. Those predicate score can be the second data for network model. As the train data is not equal to test data because the second train data is got by network model which is trained by the data itself. To make the nature of train and test data same we split data into $3[10]$.

\section{PROPOSED SYSTEM}

This automated navigation system is used to capture the image sequential via Bluetooth camera. To capture the eye direction and the eye movement correctly, the images are captured by the fixing the camera in the user's spec glass. Captured image is then integrated with raspberry pi 3 model b high detection kit. The image is processed using CNN and KNN neural networks algorithm to find out the correct accuracy in the eye position of the user [11]. This navigation system consumes less power. The pi model b has an input pin, wireless keyboard, mouse, 64 bit processor, $16 \mathrm{~GB}$ memory and 32 bit external memory. Optical cable connector port is used for wired or wireless connection. This whole process is controlled by the pi processor. Based on the eye pupil detection the system decides the direction desired by the user and direct the wheelchair automatically. If any obstacle is detected in the wheelchair root path the system automatically stop the motorized wheelchair.

There are four main operations in CNN network:

$\bullet$

- $\quad$ non linearity(ReLU)

- $\quad$ pooling or sub sampling

- $\quad$ classification(fully connected layer)

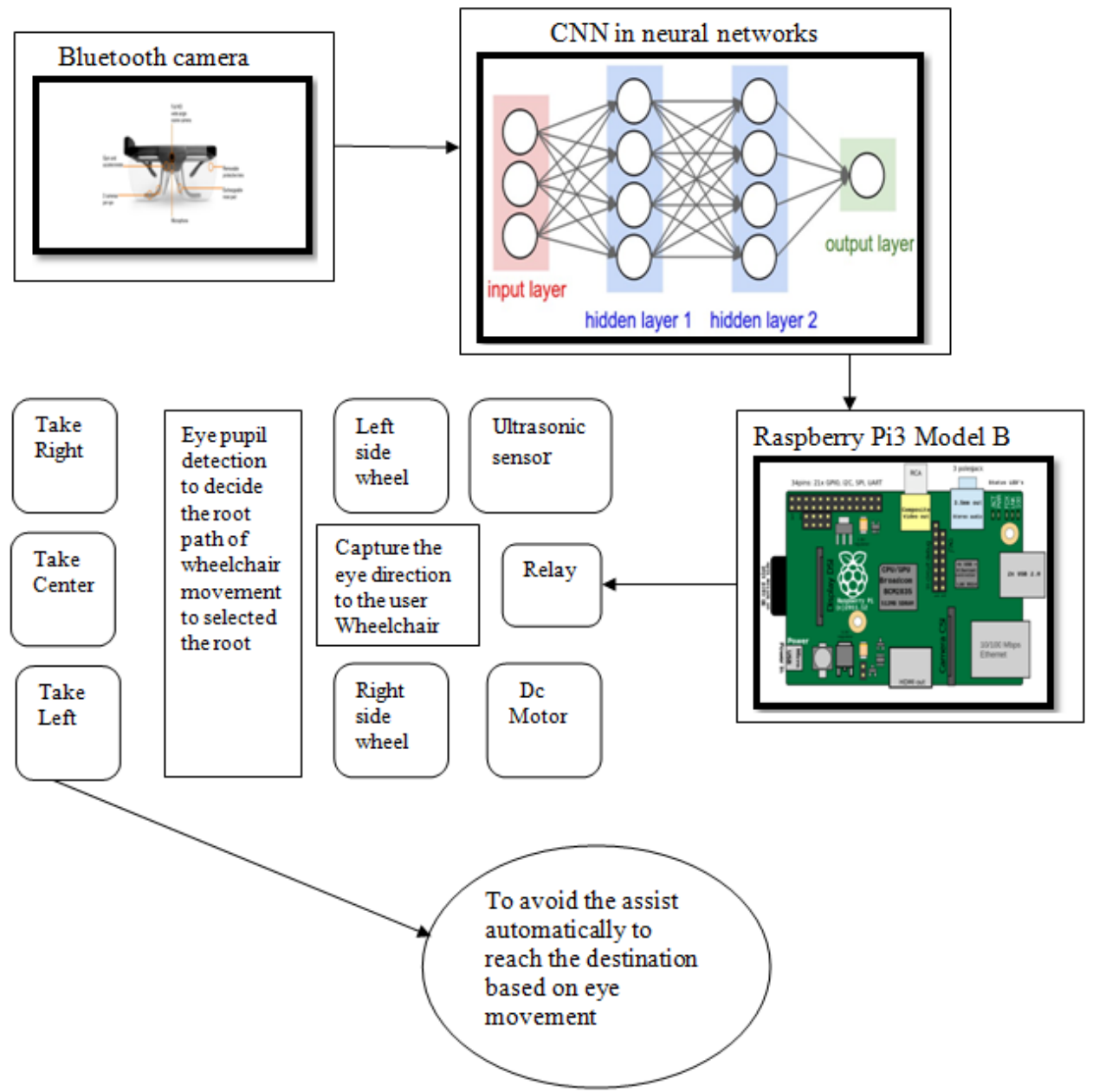

Fig. 2 block diagram for proposed method 
Vol. 8, Issue 1, January 2019

Fig.2 shows the block diagram for the proposed method in the automated eye direction safety navigation system for physically challenged person and elderly person using CNN networks. Relay is an electromechanical device. Relay is used in this navigation system to change the wheelchair root path direction of motor very fast without using the finger. Putty is an open source software the terminal emulator and network remote access to using the file transfer the application. Putty software is used to connect the remotely to raspberry pi board. Two $12 \mathrm{v}$ DC motor is used in project to demonstrate running of wheelchair in forward, reverse, left and right direction [12]. Ultrasonic sensor is a very affordable proximity / distance sensor that has been used mainly for object avoidance in various robotics projects.

\section{RESULTS AND DISCUSSION}

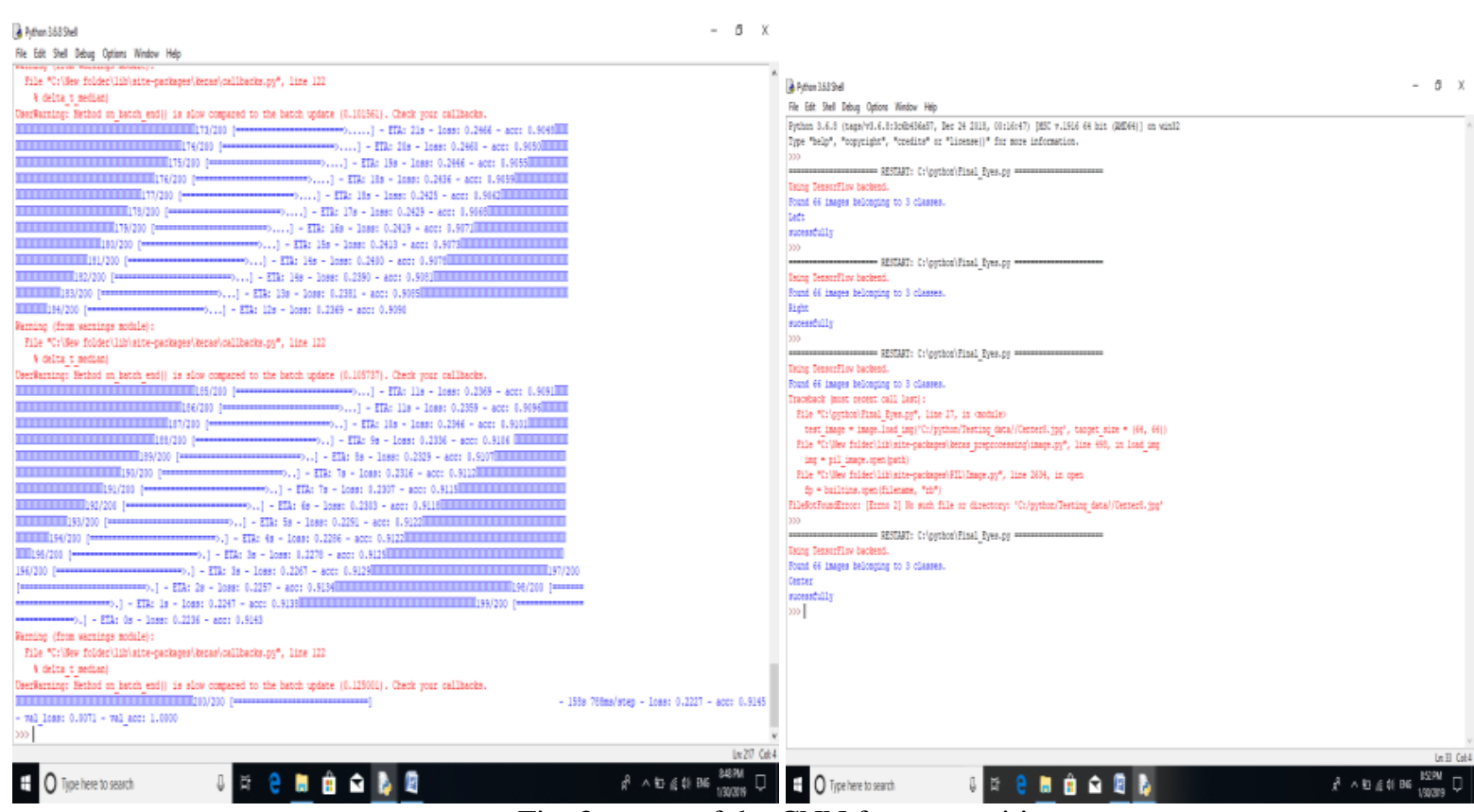

Fig. 3 output of the CNN for eye position

Fig. 3 shows that the output results of $\mathrm{CNN}$ for detect the eye position. We have taken the 5000 eye data to train and test the dataset using convolution neural network. Three types of data set train this module centre, right, left sides of the eye position and the dataset to test which type of a module.

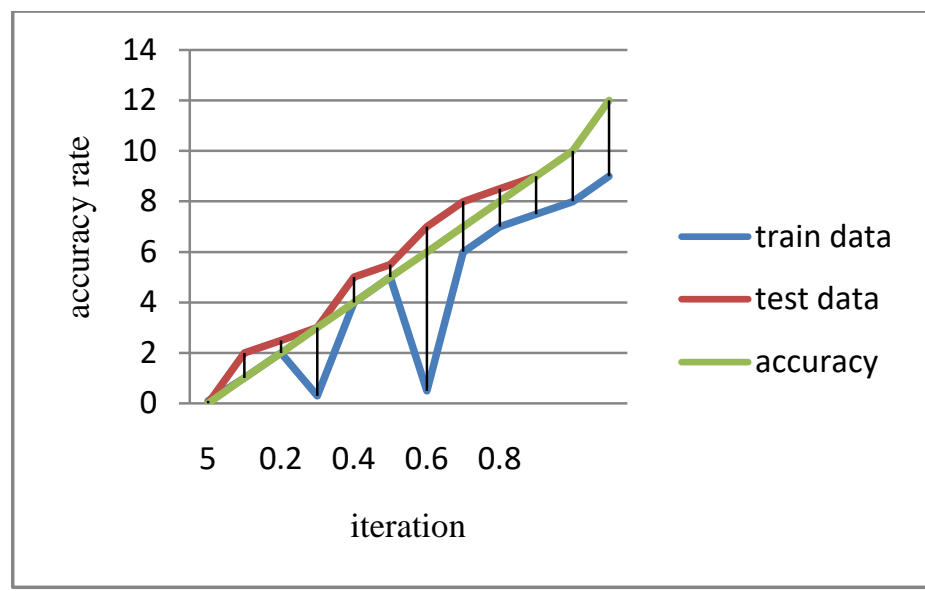

Fig. 4 eye data accuracy graph model using CNN

Fig.4 shows the classification accuracy of training and test data. The training data mark by the blue colour of the iteration position. And the test data is used to a test the training images in the test module. Test data marked by red colour. 
Vol. 8, Issue 1, January 2019

\section{CONCLUSION AND FUTURE WORK}

\section{A. Conclusion}

This proposed system is used for physically challenged people to avoid the assist. This navigation system is easy to use and operate the motorized wheelchair. This navigation system is independent and the user can choose their own direction. Bluetooth camera captures the image sequentially. Android speech to text app is used to recognize the text in the raspberry pi model $\mathrm{B}$. The system navigates the user to desired own directions such as move towards left, move towards right, move forward and stop. Android app is employed to avoid the faulty navigation in case if the person is a blind person. The limitation of project is that there may be some delay time in wheelchair movement and may be difficult to work on dark light because it would be difficult to trace the eye pupil movement.

B. Future Work

To make the system to support the recognition by voice, a voice recognition module for the blind person is also used in this motorized wheelchair. Speech to text and text to speech module can be built in python language to reduce the delay time. Some special sensors can be added to sense the direction more accurately.

\section{ACKNOWLEDGMENT}

I feel elated to keep on record our heartfelt thanks and gratitude to our project guide, Dr.V.Mohanraj, M.E,Ph.D who has been our steadfast inspiration by providing valuable guidance, his untiring patience and diligent encouragement during the entire span of this project.

\section{REFERENCES}

[1]. Parikh, Sarangi P., Valdir Grassi Jr, Vijay Kumar, and Jun Okamoto Jr. "Integrating human inputs with autonomous behaviors on an intelligent wheelchair platform." IEEE Intelligent Systems 22, no. 2 (2007): 33-41

[2]. M. Everingham,L. Van-Gool, C.K.I. Williams, J. Winn, and A. Zisserman, "The Pascal visual Object classes challenges", Ijcv, vol.88, no. 2,pp.303-338, 2010.

[3]. Y.Lihua ,D. Qi, andG. Yanjun, "Study on KNN Text Categorization Algorithm,” micro comput.info., no.21,pp.269-271,2006.

[4]. Hareesh, D., R. Raja Kishore, and K. Satyavathi. "Sensors Based Automated Wheelchair." IJRCCT 2, no. 9 (2013): 813-818

[5]. Britto, Preethika, J. Indumathi, Sudesh Sivarasu, and Lazar Mathew. "Automation of wheelchair using ultrasonic and body kinematics." In National Conference on Computational Instrumentation (NCCI2010), pp. 19-20. 2010.

[6]. Karthikeyan, T. "Efficient bio metric iris recognition system using fuzzy neural network." International Journal of Advanced Networking and Applications 1, no. 06 (2010): 371-376.

[7]. Bezdek, J. C., J. Keller, R. Krishnapuram, and N. R. Pal. "Fuzzy Models and Algorithms for Pattern Recognition and Image Processing.-NY: Springer Science+ Business Media." (2005).

[8]. Saha, Punam K., Jayaram K, Udupa, and Dewey odhner"scale based fuzzy connected image segmentation theory, algorithm, and validation". 77.2(2000) 145-174.

[9]. Analytics Vidya (website for python and (CNN)

[10]. Sinyukov, Dmitry A., Ran Li, Nicholas W. Otero, Runzi Gao, and Taşkın Padir. "Augmenting a voice and facial expression control of a robotic wheelchair with assistive navigation." In Systems, Man and Cybernetics (SMC), 2014 IEEE International Conference on, pp. 1088-1094. IEEE, 2014.

[11]. marubon-ds.blogspot.com/2017/06/title.html.

[12]. www.quro.com/is-there-a-difference-neural-networks-and-convolutional-neural-networks. 